\title{
A case report of Stevens-Johnson syndrome and toxic epidermal necrolysis due to diclofenac sodium
}

\section{Sreenivasa Rao Bendi*, Tarun Kumar Suvvari}

Department of Pharmacology, Rangaraya Medical College, Kakinada, Andhra Pradesh, India

Received: 14 May 2020

Accepted: 11 June 2020

\section{*Correspondence:}

Dr. Sreenivasa Rao Bendi,

Email: evergreenbends@gmail.com

Copyright: (C) the author(s), publisher and licensee Medip Academy. This is an open-access article distributed under the terms of the Creative Commons Attribution Non-Commercial License, which permits unrestricted non-commercial use, distribution, and reproduction in any medium, provided the original work is properly cited.

\begin{abstract}
Stevens-Johnson syndrome (SJS) is a severe skin reaction most often triggered by particular drugs in most of the cases. A more severe form of the condition is called toxic epidermal necrolysis (TEN) which involves more than $30 \%$ of the skin surface and extensive damage to the mucous membranes. SJS and TEN previously were thought to be separate conditions, but they are now considered part of a disease spectrum. The main drugs which induce SJS were anti-gout drugs, anti-epileptics, analgesics, NSAIDs and antibiotics. Diclofenac which is a NSAID and phenyl acetic acid derivative that rarely causes SJS. Although diclofenac induced Stevens-Johnson syndrome is reported very rare among adults, it shouldn't be neglected. In this report we mentioned about the Stevens-Johnson syndrome (SJS) which was later developed into TEN due to usage diclofenac sodium, in a 65 years old female patient.
\end{abstract}

Keywords: Diclofenac sodium, Stevens-Johnson syndrome, Toxic epidermal necrolysis

\section{INTRODUCTION}

Stevens-Johnson syndrome (SJS) and toxic epidermal necrolysis (TEN) are severe adverse drug reactions most commonly triggered by medications, that causes skin tissue to die (necrosis) due to detachment of epidermis from dermis. The Stevens-Johnson syndrome is an immune complex mediated type-IV hypersensitivity reaction that that typically involves the skin and the mucous membranes. ${ }^{1}$ SJS and TEN previously were thought to be separate conditions, but they are now considered part of a disease spectrum. SJS is at the less severe end of the spectrum, and TEN is at the more severe end. ${ }^{2}$

If a minor form of toxic epidermal necrolysis, with less than $10 \%$ body surface area (BSA) detachment occurs then it is classified as Stevens-Johnson syndrome (SJS). If detachment of more than $30 \%$ of the body surface area (BSA) occurs then it is classified as toxic epidermal necrolysis (TEN). The skin detachment involving 10$30 \%$ of the body surface is classified as overlapping Stevens-Johnson syndrome/toxic epidermal necrolysis. All the 3 forms are serious systemic disorders and medical emergency that can be life-threatening., ${ }^{1,2}$

SJS/TEN is a rare disease, affecting 1 to 2 per million people each year. Stevens-Johnson syndrome (the less severe form of the condition) is more common than toxic epidermal necrolysis. People who are HIV-positive and those with a chronic inflammatory disease called systemic lupus erythematosus are more likely to develop SJS/TEN than the general population. The reason for the increased risk is unclear, but immune system factors and exposure to multiple medications may play a role. $^{2}$

The initial symptoms are fever, sore throat, cough, headache, chills. Within few days a red or purplish rash form, and then the skin begins to blister and peel, leading 
to 'raw' areas of skin that are painful. In many patients, it also causes damage of oral, nasal, eye, lower respiratory tract, gastrointestinal, urethral and vaginal mucous membranes. ${ }^{1,2}$

In $25-50 \%$ of cases, Stevens-Johnson syndrome is reported as idiopathic. In adults and elderly people, the most common causes are drugs and malignancies. Pediatric cases are related more often to infections. The etiological factors are classified into 4 types - idiopathic, infectious, drug induced and malignancy related. Out of above four, the drugs are most frequently associated with SJS/TEN. The drugs include anti-gout medications (allopurinol), anti-convulsant (carbamazepine, lamotrigine, phenytoin, valproic acid), anti-retroviral drugs (nevirapine, indinavir), analgesics, NSAID's, antibiotics (mainly ciprofloxacin, penicillin's and sulfa drugs). People that may be at increased risk to develop SJS/TEN include those with HIV, a weakened immune system, a personal or family history of the condition, and certain variations of a gene called HLA-B..$^{2,3}$

An idiosyncratic, delayed hypersensitivity reaction has been implicated in the pathophysiology of StevensJohnson syndrome. Both SJS and TEN are characterized by the detachment of epidermis from the papillary dermis at the epidermal-dermal junction, manifesting as a papulomacular rash and bullae as a result of keratinocyte apoptosis. Keratinocyte apoptosis mediated by cytotoxic T-lymphocytes (CD8) in SJS and TENS is modulated by plasma tumor necrosis factor alpha and interferongamma, which are increased in patients with SJS and TEN. This process is currently hypothesized to occur through 3 possible pathways Fas-Fas ligand interaction; perforin/granzyme B; and granulysin-mediated. Progress in delineating the pathophysiology of SJS/TEN has addressed the possible risk factors interacting with natural killer and cytotoxic T-cells, such as drug structure and metabolism (CYP), and the immunogenic characteristics of the human leukocyte antigen molecules. $^{4}$

The differential diagnosis of the Stevens-Johnson syndrome (SJS) include Staphylococcal scalded skin syndrome, progressive systemic sclerosis (scleroderma), toxic shock syndrome, paraneoplastic pemphigus, epidermolysis bullosa acquisita etc. ${ }^{1}$ Management of patients with Stevens-Johnson syndrome is usually provided in emergency medicine (ICU's) and dermatology (burn centers) departments. No specific treatment of Stevens-Johnson syndrome is noted, so most of the patients are treated symptomatically. Patients should be treated with special attention to airway and hemodynamic stability, fluid status, wound/burn care, and pain control. ${ }^{4}$

Diclofenac which is a NSAID and phenyl acetic acid derivative that rarely causes Stevens-Johnson syndrome (SJS). The common symptoms of adverse drug reactions due to diclofenac are cardiac, gastrointestinal, hepatic, renal impairment. The skin reactions are very rare due to diclofenac.

This case was a Stevens-Johnson syndrome (SJS) which was later developed into toxic epidermal necrolysis (TEN). It was identified and reported during pharmacovigilance program being carried out in our institution. This case was observed with prior consent from patient and concerned authorities of Rangaraya Medical College, Kakinada.

\section{CASE REPORT}

A 65 year's old woman presented with multiple eruptions in oral cavity and on eyelids, multiple erythematous and hyper pigmented patches on the body was admitted in dermatology ward, government general hospital, Kakinada (Figure 1 and 2). Before admission in the hospital she had suffered with fever and body pains and was given tablet paracetamol and tablet cetirizine for fever. Later she was given injection diclofenac sodium for body pains. Then she developed fluid filled vesicles on the body which increased in severity and diagnosed as Stevens-Johnson syndrome (SJS).

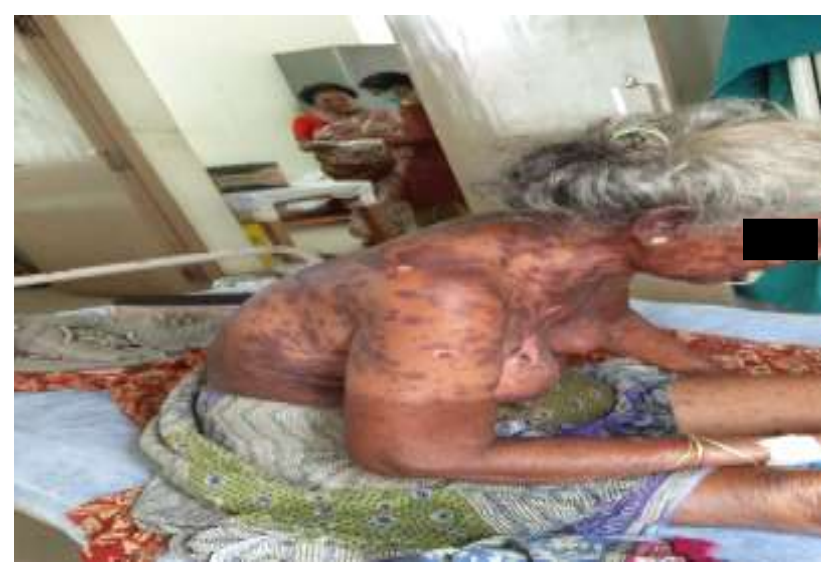

Figure 1: Multiple erythematous and hyper pigmented patches on the body.

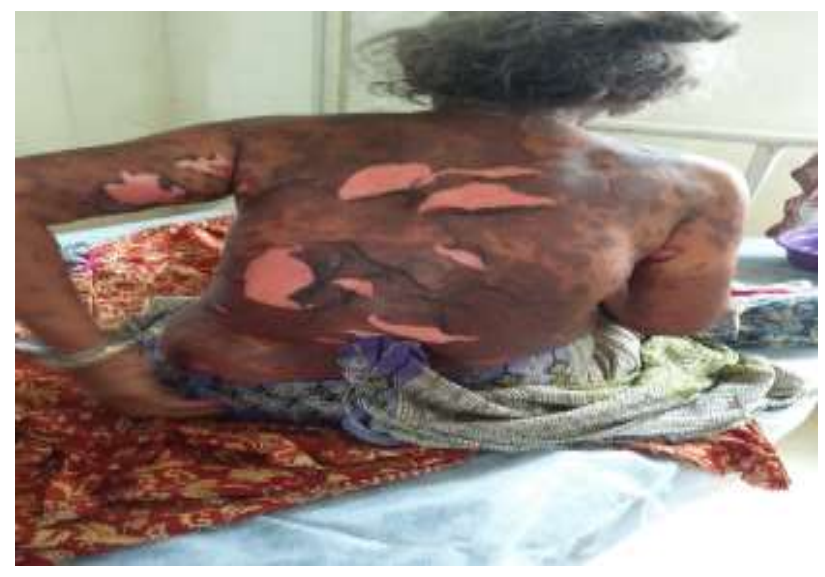

Figure 2: Blistered and peeled skin on back of the patient. 
The lesions of size varying from $0.5 \mathrm{x} 1 \mathrm{~mm}$ to $3 \mathrm{x} 2 \mathrm{~mm}$ on upper eyelids. Multiple erythematous hyper pigmented patches of $0.3 \times 0.2 \mathrm{~cm}$ to $3 \times 2 \mathrm{~cm}$ on trunk and limbs including palms. The lesions and patches had covered more than $30 \%$ of body surface area (BSA). Later they were coalesced and vesicles ruptured. The genital organs were not affected. Skin Biopsy was performed and results showed epidermal cell necrosis and infiltration of lymphocytes. The serum levels of the patient had shown elevated WBC cells and C-reactive protein.

The patient was visited by a team of specialists including dermatologist and ophthalmologist and diagnosed as Stevens-Johnson syndrome developed into toxic epidermal necrolysis. De-challenge was done. She was treated with immunoglobins, injection decadron, intravenous fluids and supportive treatment. Causality assessment was done using WHO scale. The erythematous rash with desquamation of skin was considered as a probable adverse reaction due to diclofenac sodium. The reaction subsided gradually. Rechallenge was not done. This reaction was reported to ADR monitoring center and uploaded through vigi-flow with Worldwide unique no: 2017-25197. She had recovered gradually and discharged from hospital.

\section{DISCUSSION}

In many case reports and research studies, more than 100 drugs have been implicated as causes of Stevens-Johnson syndrome or toxic epidermal necrolysis. A limited number of drugs, including sulfonamides, anticonvulsant agents, and allopurinol, are the most consistently associated with these conditions and nonsteroidal antiinflammatory drugs (NSAIDs), analgesic agents, and non-sulfonamide antibiotics are associated with significant but lower risks for SJS and TEN. The relative risk associated with the use of specific drugs has never been quantified. The risk of adverse drug reactions due to diclofenac sodium was found to be more associated in the females than males. ${ }^{5,7}$

Today, drug therapy for the control of pain has been encountered a great problem because of drugs serious adverse reactions. In a study the upper 95 percent confidence limits for propionic acid derivatives and for diclofenac make it impossible to rule out some increase in risk. ${ }^{6}$ As the patient showed positive response after dechallenge, this implies the contribution of diclofenac to the skin reaction. So vigilant administration should be done in case of diclofenac sodium.

\section{CONCLUSION}

Stevens-Johnson syndrome and toxic epidermal necrolysis are acute life-threatening conditions. Although non-steroidal anti-inflammatory drugs (NSAIDs) are a rare cause of SJS in adults, these risks should be not ignored. This risk is higher for older patients, women and those in the beginning of the treatment. Stevens-Johnson syndrome is a very rare complication of diclofenac use but due to its dangerous consequences, practitioners should be aware of it and give information to their patients especially if patients are old age people and women.

\section{Funding: No funding sources \\ Conflict of interest: None declared \\ Ethical approval: Not required}

\section{REFERENCES}

1. Foste CS. Stevens-Johnson Syndrome. Medscape Reference 17 Jan 2019. Available at https://emed icine.medscape.com/article/1197450-overview. Accessed on 10 May 2020.

2. Stevens-Johnson syndrome/toxic epidermal necrolysis. Genetics Home Reference (GHR). Available at https://ghr.nlm.nih.gov/condition/ stevens-johnson-syndrome-toxic-epidermalnecrolysis. Accessed on 10 May 2020.

3. Stevens-Johnson syndrome. Mayo Clinic Reference. Available at https://www.mayoclinic.org/diseasesconditions/stevens-johnson-syndrome/symptomscauses/syc-20355936. Accessed on 10 May 2020.

4. Stevens-Johnson Syndrome. Epocrates Reference. Available at https://online.epocrates.com/diseases/ 23724/Stevens-Johnson-syndrome/Etiology. Accessed on 10 May 2020.

5. Babamahmoodi F, Eslami G, Babamahmoodi A. Diclofenac-Induced Stevens-Johnson Syndrome: A Case Report. Iranian J Pharmacology Therapeutics. 2012;11(1):33-5.

6. Roujeau JC, Kelly JP, Naldi L, Rzany B, Stern RS, Anderson T, et al. Medication use and the risk of Stevens-Johnson syndrome or toxic epidermal necrolysis. New England J Med. 1995;333(24):16008.

7. Roujeau JC, Flahault A. Stevens-Johnson syndrome and toxic epidermal necrolysis: assessment of medication risks with emphasis on recently marketed drugs, the Euro SCAR study. J Investigative Dermatol. 2008;128(1):35-44.

Cite this article as: Bendi SR, Suvvari TK. A case report of stevens: Johnson syndrome and toxic epidermal necrolysis due to diclofenac sodium. Int $\mathbf{J}$ Basic Clin Pharmacol 2020;9:1132-4. 